Bundesgesundheitsbl 2013 · 56:802-808

DOI 10.1007/s00103-013-1698-1

Online publiziert: 27. Mai 2013

(c) Springer-Verlag Berlin Heidelberg 2013

\section{Additional material online}

An English full-text version of this article is available at SpringerLink under supplementary material: dx.doi.org/10.1007/s00103-013-1698-1

T. Lampert · E. von der Lippe · S. Müters

Abteilung für Epidemiologie und Gesundheitsmonitoring, Robert Koch-Institut, Berlin

\title{
Verbreitung des Rauchens in der Erwachsenenbevölkerung in Deutschland
}

\section{Ergebnisse der Studie zur Gesundheit Erwachsener in Deutschland (DEGS1)}

Arbeitsstättenverordnung im Jahr 2002, die Heraufsetzung der Altersgrenze für den Kauf und Konsum von Tabakprodukten im Jahr 2008, die Einschränkung bzw. das weitgehende Verbot von Tabakwerbung entsprechend des geltenden EU-Rechts sowie die seit 2007 erlassenen Nichtraucherschutzgesetze des Bundes und der Länder, die sich auf öffentliche Gebäude und Verkehrsmittel, Schulen und Krankenhäuser sowie auf gastronomische Betriebe beziehen. Unterstützt werden diese durch bundesweite Aufklärungskampagnen der Bundeszentrale für gesundheitliche Aufklärung (BZgA) und settingbezogene Präventionsmaßnahmen, die unter anderem in Schulen, Betrieben und Krankenhäusern umgesetzt werden [3, 7].

Der vorliegende Beitrag befasst sich auf Basis von Daten der Studie zur Gesundheit Erwachsener in Deutschland (DEGS1) mit folgenden Fragestellungen: 1) Wie verbreitet ist das Rauchen in der 18- bis 79-jährigen Bevölkerung Deutschlands? 2) Inwieweit sind altersund geschlechtsspezifische Unterschiede im Rauchverhalten zu beobachten? 3) Besteht ein Zusammenhang zwischen dem sozialen Status und dem Rauchen und wie stark ist dieser ggf. ausgeprägt? 4) Wie hat sich das Rauchverhalten in den letzten 20 Jahren entwickelt?

\section{Methode}

Die „Studie zur Gesundheit Erwachsener in Deutschland“ (DEGS) ist Bestandteil des Gesundheitsmonitorings des Robert Koch-Instituts (RKI). Konzept und Design von DEGS sind an anderer Stelle ausführlich beschrieben $[8,9,10,11,12]$. Die erste Erhebungswelle (DEGS1) wurde von 2008 bis 2011 durchgeführt und umfasste Befragungen, Untersuchungen und Tests $[13,14]$. Zielpopulation war die in Deutschland lebende Bevölkerung im Alter von 18 bis 79 Jahren. DEGS1 hat ein Mischdesign, das gleichzeitig querund längsschnittliche Analysen ermöglicht. Hierbei wurde eine Einwohnermeldeamtsstichprobe durch ehemalige Teilnehmerinnen und Teilnehmer des Bundes-Gesundheitssurveys 1998 (BGS98) ergänzt. Insgesamt nahmen 8152 Personen teil, darunter 4193 Ersteingeladene (Response 42\%) und 3959 ehemalige Teilnehmerinnen und Teilnehmer des BGS98 (Response 62\%). 7238 Personen besuchten eines der 180 Untersuchungszentren, 914 wurden ausschließlich befragt. Die Nettostichprobe [12] ermöglicht für den Altersbereich von 18 bis 79 Jahren repräsentative Querschnittanalysen und Trendaussagen im Vergleich mit dem BGS98 ( $\mathrm{n}=7988$, davon 7116 in Untersuchungszentren). Die Daten der erneut Teilnehmenden sind für Längsschnittanalysen nutzbar. Die Querschnitt- und Trendanalysen werden mit einem Ge- 


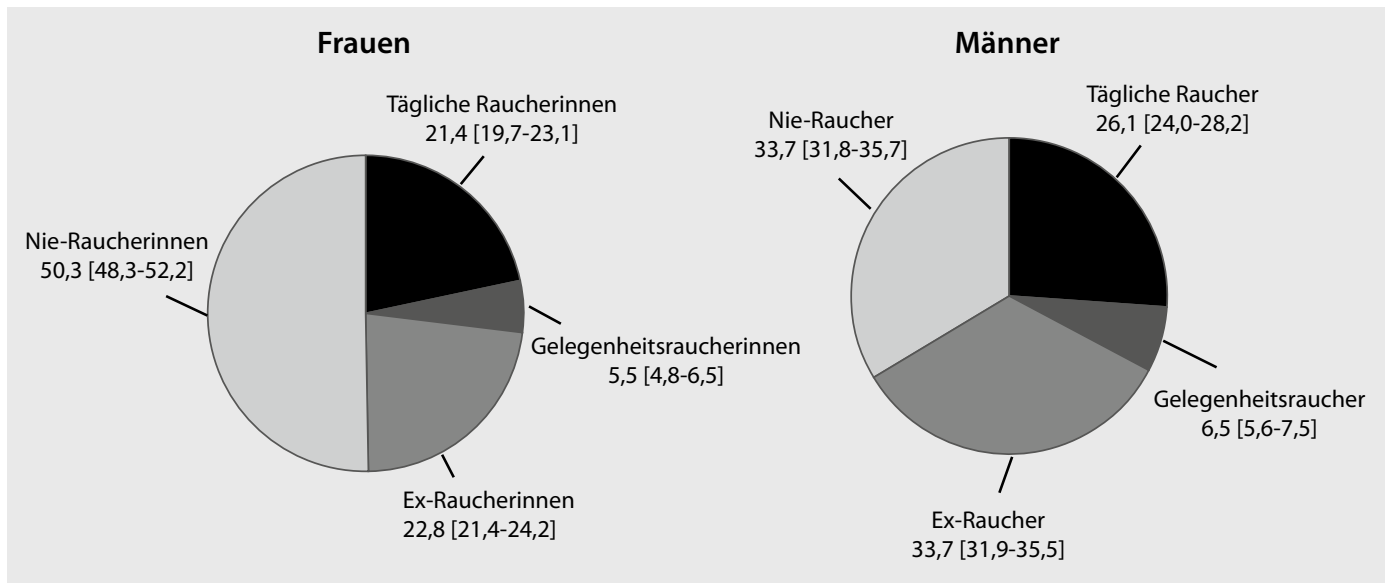

Abb. $1<$ Verbreitung des Rauchens bei 18- bis 79jährigen Frauen und Männern. Dargestellt sind Prävalenzen (in \%) und 95\%Konfidenzintervallen $(n=7899)$

wichtungsfaktor durchgeführt, der Abweichungen der Stichprobe von der Bevölkerungsstruktur (Stand 31.12.2010) hinsichtlich Alter, Geschlecht, Region und Staatsangehörigkeit sowie Gemeindetyp und Bildung korrigiert [12]. Für den Untersuchungsteil wurde ein gesonderter Gewichtungsfaktor erstellt. Bei der Berechnung für die ehemaligen Teilnehmenden des BGS98 wurde die Wiederteilnahmewahrscheinlichkeit, basierend auf einem logistischen Modell, berücksichtigt. Für die Durchführung von Trendanalysen werden die Daten des Bundes-Gesundheitssurveys 1998 auf den Bevölkerungsstand zum 31.12.2010 altersadjustiert. Eine Nonresponder-Analyse und der Vergleich einzelner erhobener Indikatoren mit Daten der amtlichen Statistik weisen auf eine hohe Repräsentativität der Stichprobe für die Wohnbevölkerung in Deutschland hin [12].

Im Folgenden werden vor allem Prävalenzen mit 95\%-Konfidenzintervallen berichtet. Neben alters- und geschlechtsspezifischen Unterschieden wird auch auf Unterschiede nach dem sozialen Status eingegangen. Der Sozialstatus wurde anhand eines Indexes bestimmt, in den Angaben zu schulischer und beruflicher Ausbildung, beruflicher Stellung sowie Haushaltsnettoeinkommen (bedarfsgewichtet) eingehen und der eine Einteilung in niedrige, mittlere und hohe Statusgruppe ermöglicht $[15,16]$. Um sowohl die Gewichtung als auch die Korrelation der Teilnehmenden innerhalb einer Gemeinde zu berücksichtigen, wurden die Konfidenzintervalle mit den Verfahren für komplexe Stichproben von SPSS-20 bestimmt. Unterschiede werden als sta- tistisch signifikant angesehen, wenn sich die 95\%-Konfidenzintervalle nicht überschneiden. Um Veränderungen im Zeitverlauf beurteilen zu können, werden zusätzlich p-Werte ausgewiesen, die mithilfe von Regressionsanalysen, in denen die Veränderung der Altersstruktur als erklärende Variable berücksichtigt wird, berechnet wurden. Die p-Werte, die damit aussagen, ob eine von diesem Alterseffekt unabhängige Veränderung des Rauchverhaltens stattgefunden hat, wurden ebenfalls mit den SPSS-20-Verfahren für komplexe Stichproben ermittelt.

Das Rauchverhalten wurde mit mehreren Fragen erhoben, die sich unter anderem auf das aktuelle bzw. frühere Rauchverhalten sowie die Intensität des Rauchens beziehen. Zunächst sollte die Frage beantwortet werden: „Rauchen Sie zurzeit - wenn auch nur gelegentlich?" (Antwortkategorien: „Ja, täglich“, „Ja, gelegentlich“, „Nein, nicht mehr“, „Habe noch nie geraucht"). Anhand der Antworten auf diese Frage wird im Folgenden zwischen „Täglichen Raucherinnen bzw. Rauchern“, „Gelegenheitsraucherinnen bzw. -rauchern“, „Ex-Raucherinnen bzw. -Rauchern“, „Nie-Raucherinnen bzw. -Rauchern“ unterschieden. Wenn von aktuellen Raucherinnen bzw. Rauchern die Rede ist, werden die täglichen und gelegentlichen Raucherinnen bzw. Raucher zusammen betrachtet. Darüber hinaus werden Angaben zu den „Jemalsraucherinnen bzw. -rauchern (tägliche, gelegentliche und ehemalige Raucherinnen bzw. Raucher)“ und zur „Ausstiegsquote (Anteil der ehemaligen Raucherinnen bzw. Raucher an den Jemalsraucherinnen bzw. -rauchern)“ gemacht.
Um möglichst genaue Aussagen über die Intensität des Rauchens treffen zu können, sollten die Befragten angeben, wie viel sie durchschnittlich am Tag (tägliche Raucherinnen bzw. Raucher) bzw. in der Woche (Gelegenheitsraucherinnen bzw. -raucher) rauchen, wobei zwischen fabrikfertigen Zigaretten, selbstgedrehten oder selbstgestopften Zigaretten, Zigarren/Zigarillos, Pfeifen sowie Wasserpfeifen unterschieden wurde. Im Folgenden wird der Anteil der starken Zigarettenraucherinnen bzw. Zigarettenraucher betrachtet. Gemäß einer Einschätzung der Weltgesundheitsorganisation (WHO) sind dies die Personen, die 20 und mehr Zigaretten am Tag rauchen [17].

Für die Analyse zeitlicher Entwicklungen und Trends des Rauchverhaltens wurden zusätzlich die Daten des gepoolten Datensatzes für den Nationalen Gesundheitssurveys 1990-91 und den Gesundheitssurvey OST 1991-92 (NUS9092, $\mathrm{n}=7407)$, des Bundes-Gesundheitssurveys 1998 (BGS98, n=7124), des telefonischen Gesundheitssurveys 2003 (GSTel03, n=8318) und der Studie Gesundheit in Deutschland aktuell 2009 (GEDA09, n=21.262) herangezogen. In diesen Gesundheitssurveys, die allesamt vom Robert Koch-Institut durchgeführt wurden, erfolgte die Erhebung des Rauchverhaltens in vergleichbarer Weise wie in DEGS1. Für die langfristige Analyse, die bis zum Beginn der 1990er-Jahre zurückreicht, muss allerdings eine Eingrenzung auf die 25- bis 69-Jährigen erfolgen, da der NUST2 und der SURVEY OST auf diese Altersspanne beschränkt waren. Für den Vergleich der Ergebnisse von DEGS1 mit denen des BGS98, des 
GSTel03 und GEDA09 kann der Altersbereich von 18 bis 79 Jahren betrachtet werden. $\mathrm{Zu}$ berücksichtigen ist außerdem, dass sich die Gesundheitssurveys in Bezug auf das Studiendesign, die Teilnahmebereitschaft und die Stichprobenzusammensetzung unterscheiden. So handelt es sich beim GSTel03 und GEDA09 um telefonische Befragungen mit einer Response von 31 bzw. 29\% [18], während die anderen Surveys als kombinierte Befragungs- und Untersuchungssurveys in Studienzentren vor Ort durchgeführt wurden, wobei die Response zwischen $62 \%$ im BGS98 und $42 \%$ in DEGS1 variierte. Für die Trendanalysen wurden zwar spezielle Gewichtungsfaktoren berechnet, diese können die Heterogenität der Surveys aber allenfalls teilweise ausgleichen [19].

\section{Ergebnisse}

Nach den Daten der DEGS-Studie rauchen $29,7 \%$ der 18- bis 79-jährigen Erwachsenen täglich oder gelegentlich. Frauen rauchen $\mathrm{zu}$ 26,9\% und damit $\mathrm{zu}$ einem geringeren Anteil als Männer, die zu 32,6\% zumindest gelegentlich zur Zigarette oder einem anderen der betrachteten Tabakprodukte greifen. Weitere 22,8\% der Frauen und 33,7\% der Männer haben früher geraucht, mittlerweile das Rauchen aber aufgegeben. Dass sie nie geraucht haben, trifft auf die Hälfte der 18- bis 79-jährigen Frauen und ein Drittel der gleichaltrigen Männer zu ( $\bullet$ Abb. 1).

Am häufigsten wird im jungen Erwachsenenalter geraucht. Bei 18- bis 29-jährigen Frauen beträgt die Prävalenz für das aktuelle Rauchen (täglich oder gelegentlich) $40,0 \%$, bei gleichaltrigen Männern 47,0\%. Im mittleren Lebensalter liegen die Prävalenzen um die 30\% bei Frauen und zwischen 30 und $40 \%$ bei Männern. Von den Frauen und Männern im Alter von 65 bis 79 Jahren rauchen 8,9 bzw. 11,6\% und damit ein geringerer Anteil als im jungen und mittleren Erwachsenenalter (• Tab. 1).

Etwa ein Viertel der Frauen und Männer, die rauchen, konsumieren 20 und mehr Zigaretten am Tag. Bezogen auf die Gesamtbevölkerung im Alter von 18 bis 79 Jahren kann die Prävalenz des starken Rauchens mit $8,3 \%$ beziffert werden, wo-

Bundesgesundheitsbl 2013 · 56:802-808 DOI 10.1007/s00103-013-1698-1

(c) Springer-Verlag Berlin Heidelberg 2013

\section{T. Lampert $\cdot$ E. von der Lippe $\cdot$ S. Müters \\ Verbreitung des Rauchens in der Erwachsenenbevölkerung in Deutschland. Ergebnisse der Studie zur Gesundheit Erwachsener in Deutschland (DEGS1)}

\section{Zusammenfassung}

Obwohl in Deutschland in den letzten Jahren verschiedene Maßnahmen zur Eindämmung des Tabakkonsums umgesetzt wurden, ist das Rauchen nach wie vor stark verbreitet und stellt ein erhebliches Risiko für die Gesundheit der Bevölkerung dar. Nach den Daten der Studie zur Gesundheit Erwachsener in Deutschland (DEGS1), die vom Robert KochInstitut in den Jahren 2008 bis 2011 durchgeführt wurde, rauchen $29,7 \%$ der 18 - bis 79-jährigen Erwachsenen (Frauen $=26,9 \%$, Männer $=32,6 \%)$. Der Anteil der Frauen und Männer, die 20 oder mehr Zigaretten am Tag rauchen, beträgt 6,0 bzw. $10,6 \%$. Am stärks- ten verbreitet ist das Rauchen bei jungen Erwachsenen, außerdem bei Personen mit niedrigem Sozialstatus, die auch überproportional zu den starken Raucherinnen bzw. Rauchern zu zählen sind. Der Vergleich mit den Daten früherer Gesundheitssurveys deutet darauf hin, dass der Anteil der Raucherinnen und Raucher im Verlauf der letzten 10 Jahre etwas zurückgegangen ist.

Schlüsselwörter

Tabakkonsum · Rauchen .

Gesundheitsverhalten · Soziale Ungleichheit . Gesundheitssurvey

\section{Prevalence of smoking in the adult population of Germany. Results of the German Health Interview and Examination Survey for Adults (DEGS1)}

\section{Abstract}

Although various tobacco control measures have been implemented in Germany in the recent years, smoking is still widespread and constitutes a considerable health risk for the population. According to the data of the German Health Interview and Examination Survey for Adults (DEGS1), which was conducted by Robert Koch Institute from 2008-2011, $29.7 \%$ of the 18 - to 79 -year-old population smokes ( women $=26.9 \%$, men $=32.6 \%$ ). The proportion of women and men who smoke 20 or more cigarettes a day amounts to 6.0 and $10.6 \%$ respectively. Smoking is most- ly widespread among young adults, as well as among persons with low social status who are also overrepresented among the heavy smokers. Comparison with data from previous health surveys indicates that the proportion of smokers has reduced slightly over the last 10 years. An English full-text version of this article is available at SpringerLink as supplemental.

Keywords

Tobacco consumption · Smoking $\cdot$ Health behaviour $\cdot$ Social inequality $\cdot$ Health survey bei der Wert für Frauen mit 6,0\% unter dem Vergleichswert für Männer mit $10,6 \%$ liegt.

Der Anteil der starken Raucherinnen und Raucher ist in der Altersgruppe der 30- bis 44-Jährigen am höchsten (• Abb. 2). Von den Frauen und Männer dieses Alters rauchen 8,5 bzw. 16,8\% stark. Die geringsten Anteile an starken Raucherinnen und Rauchern finden sich mit 1,5 bzw. 2,4\% in der 65- bis 79-jährigen Bevölkerung.

Darüber hinaus sind Unterschiede im Rauchverhalten nach dem sozialen Status festzustellen (• Abb.3). Frauen und Männer mit niedrigem Sozialstatus rauchen etwa zweimal häufiger als Frau- en und Männer mit hohem Sozialstatus. Noch deutlicher fallen die statusspezifischen Unterschiede in Bezug auf das starke Rauchen aus, und zwar insbesondere bei Männern. Außerdem zeigt sich, dass die Angehörigen der mittleren Statusgruppe im Vergleich zu denen der hohen Statusgruppe häufiger rauchen und vermehrt zu den starken Rauchern gehören. Auch gegenüber den Angehörigen der niedrigen Statusgruppe bestehen Unterschiede, die aber statistisch nicht signifikant sind.

Die statusspezifischen Unterschiede im Rauchverhalten kommen dadurch zustande, dass die Angehörigen der niedrigen Statusgruppen häufiger mit dem 
Tab. 1 Verbreitung des Rauchens bei Frauen und Männern in verschiedenen Altersgruppen. Dargestellt sind Prävalenzen (in \%) mit 95\%-Konfidenzintervallen ( $n=7899$ )

\begin{tabular}{|c|c|c|c|c|}
\hline \multirow[t]{2}{*}{ Jahre } & \multicolumn{4}{|l|}{ Rauchverhalten } \\
\hline & Täglich & Gelegentlich & Früher & $\mathrm{Nie}$ \\
\hline \multicolumn{5}{|c|}{ Frauen } \\
\hline $18-29$ & $29,7[25,7-34,1]$ & $10,3[7,8-13,5]$ & $14,5[11,5-18,0]$ & $45,5[41,0-50,1]$ \\
\hline $30-44$ & $24,6[21,2-28,3]$ & $6,6[5,0-8,6]$ & $20,4[17,5-23,5]$ & $48,5[44,4-52,5]$ \\
\hline $45-64$ & $23,2[20,4-26,2]$ & $4,7[3,6-6,1]$ & $30,3[27,7-32,9]$ & $41,9[38,9-44,9]$ \\
\hline $65-79$ & $7,1[5,5-9,3]$ & $1,8[1,1-3,1]$ & $20,0[16,7-23,7]$ & $71,1[67,0-74,8]$ \\
\hline \multicolumn{5}{|c|}{ Männer } \\
\hline $18-29$ & $34,2[29,6-39,2]$ & $12,8[10,0-16,2]$ & $12,6[9,5-16,4]$ & $40,4[35,4-45,7]$ \\
\hline $30-44$ & $32,1[28,0-36,5]$ & $7,7[5,8-10,3]$ & $24,1[20,4-28,4]$ & $36,0[31,9-40,4]$ \\
\hline $45-64$ & $25,6[22,5-29,0]$ & $4,6[3,5-6,2]$ & $43,0[40,0-46,1]$ & $26,7[24,0-29,6]$ \\
\hline $65-79$ & $9,8[7,7-12,3]$ & $1,8[1,0-3,1]$ & $50,8[47,2-54,5]$ & $37,6[34,1-41,3]$ \\
\hline
\end{tabular}

Tab. 2 Ausstiegsquote nach Alter, Geschlecht und Sozialstatus. Dargestellt sind Prävalenzen (in \%) mit 95\%-Konfidenzintervallen ( $\mathrm{n}=4510)$

\begin{tabular}{|lllll}
\hline Jahre & Gesamt & $\begin{array}{l}\text { Niedriger Sozial- } \\
\text { status }\end{array}$ & $\begin{array}{l}\text { Mittlerer Sozial- } \\
\text { status }\end{array}$ & $\begin{array}{l}\text { Hoher Sozial- } \\
\text { status }\end{array}$ \\
\hline Frauen & & & & \\
\hline $18-29$ & $26,5[21,4-32,4]$ & $26,5[16,8-39,3]$ & $25,1[18,8-32,6]$ & $35,4[20,2-54,1]$ \\
\hline $30-44$ & $39,5[34,1-43,5]$ & $29,8[19,3-42,9]$ & $36,2[30,0-42,9]$ & $57,8[45,8-68,9]$ \\
\hline $45-64$ & $52,1[48,0-56,1]$ & $36,9[27,4-47,6]$ & $51,2[46,3-56,1]$ & $67,7[59,8-74,7]$ \\
\hline $65-79$ & $69,0[62,2-75,1]$ & $59,9[41,7-75,7]$ & $70,8[62,6-77,8]$ & $78,5[64,0-88,3]$ \\
\hline Gesamt & $45,8[43,2-48,5]$ & $35,8[30,2-41,8]$ & $44,6[41,5-47,8]$ & $61,8[56,0-67,0]$ \\
\hline Männer & & & & \\
\hline $18-29$ & $21,1[16,1-27,1]$ & $17,0[8,9-29,8]$ & $19,8[14,0-27,2]$ & $41,5[23,3-62,4]$ \\
\hline $30-44$ & $37,7[32,3-43,5]$ & $28,9[18,5-42,0]$ & $33,3[26,6-40,6]$ & $56,6[45,6-67,0]$ \\
\hline $45-64$ & $58,7[54,8-62,5]$ & $49,4[40,2-58,6]$ & $58,2[53,4-62,9]$ & $69,9[63,0-75,5]$ \\
\hline $65-79$ & $81,5[77,5-84,9]$ & $75,4[63,2-84,6]$ & $83,0[77,9-87,1]$ & $85,6[78,2-90,7]$ \\
\hline Gesamt & $50,8[48,2-53,5]$ & $42,8[36,8-49,0]$ & $49,2[45,8-52,6]$ & $65,6[60,7-70,2]$ \\
\hline & & & & \\
\hline
\end{tabular}

Tab. 3 Veränderung des Rauchverhaltens bei Frauen und Männern verschiedener Altersgruppen im Zeitraum 2003 bis 2011. Dargestellt sind Prävalenzen (in \%), die Differenz zwischen beiden Beobachtungsjahren in Prozentpunkten und der $\mathrm{p}$-Wert für die ausgewiesene Differenz ( $n=8163$ für GSTel03, $n=7899$ für DEGS1)

\begin{tabular}{lllll}
\hline Jahre & $\mathbf{2 0 0 3}$ & $\mathbf{2 0 1 1}$ & Differenz & p-Wert \\
\hline Frauen & & & & \\
\hline $18-29$ & 46,2 & 40,0 & $-6,2$ & 0,068 \\
\hline $30-44$ & 39,6 & 31,2 & $-8,4$ & 0,001 \\
\hline $45-64$ & 27,8 & 27,8 & $\pm 0,0$ & 0,747 \\
\hline $65-79$ & 7,0 & 9,0 & $+2,0$ & 0,150 \\
\hline Gesamt & 29,8 & 26,9 & $-2,9$ & 0,019 \\
\hline Männer & & & & \\
\hline $18-29$ & 54,5 & 47,0 & $-7,5$ & 0,032 \\
\hline $30-44$ & 45,9 & 39,8 & $-6,1$ & 0,033 \\
\hline $45-64$ & 33,4 & 30,3 & $-3,1$ & 0,056 \\
\hline $65-79$ & 17,1 & 11,5 & $-5,6$ & 0,011 \\
\hline Gesamt & 38,8 & 32,6 & $-6,2$ & 0,000 \\
\hline
\end{tabular}

Rauchen beginnen und seltener wieder aufhören. Dies trifft zumindest auf Männer zu, während bei Frauen die Unterschiede im Ausstiegsverhalten ausschlag- gebend zu sein scheinen. Von den 18- bis 79-jährigen Männern mit niedrigem Sozialstatus haben 72,9\% mit dem Rauchen begonnen, von den Männern mit mitt- lerem und hohem Sozialstatus waren es 68,9 bzw. 54,3\%. Die Vergleichswerte für Frau en betragen 48,3\%, 50,7 und 47,7\% und weisen bezüglich des Einstiegsverhaltens auf keine statistisch bedeutsamen Unterschiede zwischen den Statusgruppen hin.

Demgegenüber sind sowohl bei Frauen als auch bei Männern Unterschiede im Ausstiegsverhalten zu beobachten. Die Ausstiegsquote, die den Anteil der ExRaucherinnen bzw. Ex-Raucher an den Personen bezeichnet, die jemals mit dem Rauchen begonnen haben, beläuft sich bei 18- bis 79-jährigen Frauen mit niedrigem Sozialstatus auf $35,8 \%$, während sie bei Frauen dieses Alters, die der hohen Statusgruppe zuzurechnen sind, 61,8\% beträgt. Bei Männern sind diese Unterschiede mit Werten von $42,8 \%$ in der niedrigen und 65,6\% in der hohen Statusgruppe ähnlich stark ausgeprägt. Eine altersdifferenzierte Betrachtung weist die statusspezifischen Variationen der Ausstiegsquote im mittleren Lebensalter als statistisch bedeutsam aus (- Tab. 2).

Aussagen über längerfristige zeitliche Entwicklungen und Trends beim Rauchen sind für die 25- bis 69-jährige $\mathrm{Be}$ völkerung möglich (• Abb. 4). Ab den 1990er-Jahren sprechen die Daten bei Frauen für einen Anstieg der Prävalenz um mehr als 5 Prozentpunkte bis auf $32,0 \%$ im Jahr 2003, während sich für Männer keine wesentlichen Veränderungen beobachten lassen. Für den Zeitraum von 2003 bis 2009 ist hingegen bei Frauen wie Männern ein Rückgang der Rauchquote festzustellen, und zwar um jeweils etwa 2 Prozentpunkte. Seitdem hat die Rauchquote bei Frauen stagniert und beläuft sich im Jahr 2011, wenn die Altersspanne von 25 bis 69 Jahre betrachtet wird, auf 29,3\%. Bei Männern hat sich der rückläufige Trend weiter fortgesetzt, sodass die Prävalenz bis zum Jahr 2011 um weitere 2 Prozentpunkte auf $34,4 \%$ sank.

Bei Frauen ist der Rückgang des Rauchens nur in den jüngeren Altersgruppen zu beobachten, bei Männern außerdem in den höheren Altersgruppen. Dies verdeutlicht der Vergleich der Jahre 2003 und 2011, für den auch die Altersgruppen der unter 25-Jährigen und der 70 bis 79-Jährigen betrachtet werden können (• Tab. 3). Als statistisch signifi- 


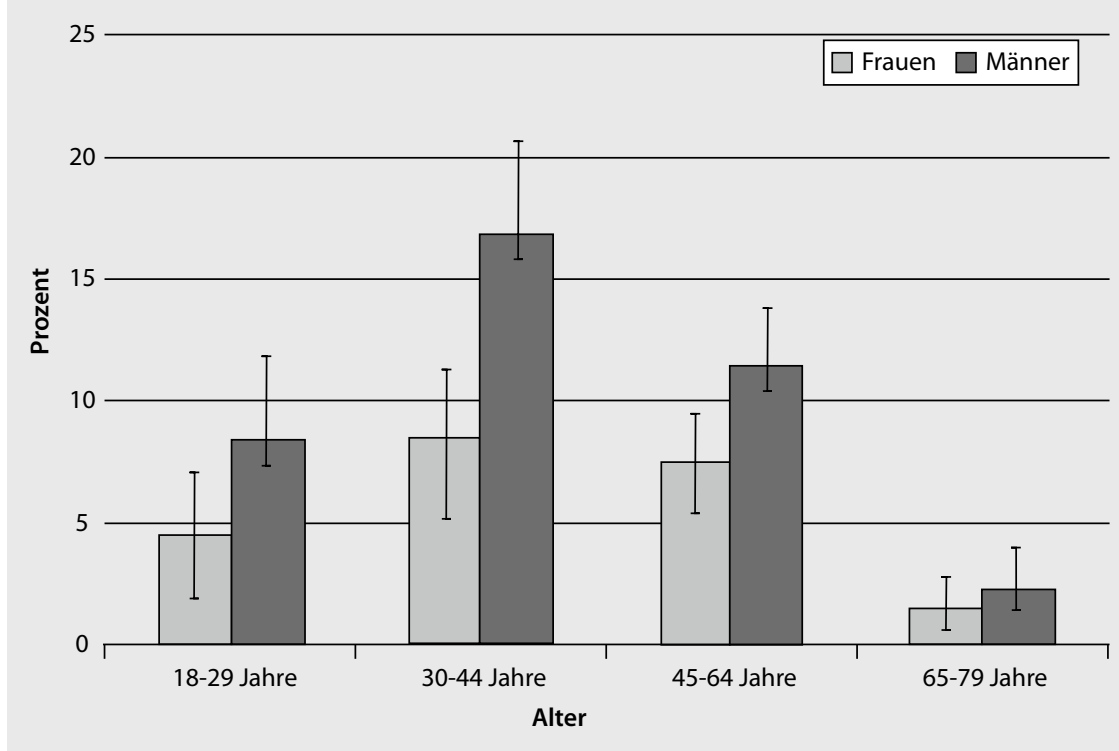

Abb. $2 \Delta$ Verbreitung des starken Rauchens bei 18- bis 79-jährigen Frauen und Männern. Dargestellt sind Prävalenzen (in \%) mit 95\%-Konfidenzintervallen ( $\mathrm{n}=7755)$

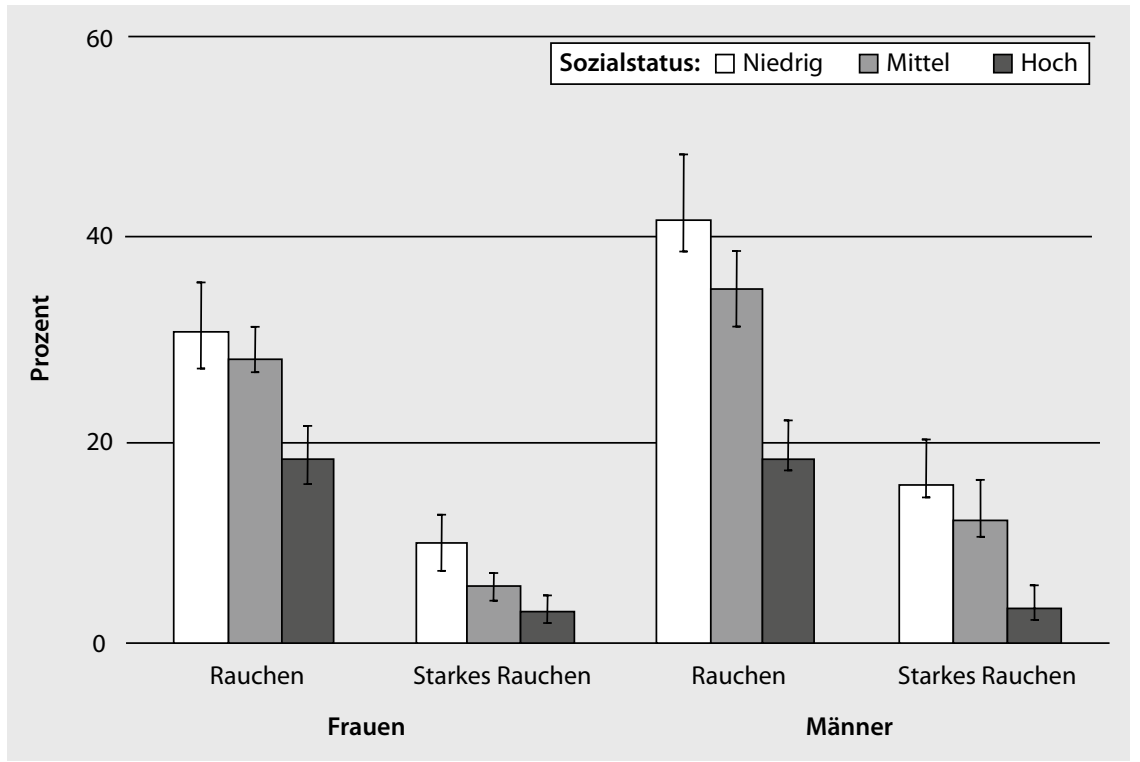

Abb. $3 \triangle$ Verbreitung des Rauchens und des starken Rauchens nach sozialem Status bei 18- bis 79jährigen Frauen und Männern. Dargestellt sind Prävalenzen (in \%) mit 95\%-Konfidenzintervallen ( $\mathrm{n}=7858$ für das Rauchen, $\mathrm{n}=7717$ für das starke Rauchen)

kant erweist sich bei Frauen die Entwicklung in der Gruppe der 30- bis 44-Jährigen. Bei Männern ist in allen Altersgruppen, mit Ausnahme der 45- bis 64-Jährigen, ein statistisch bedeutsamer Rückgang der Rauchquote festzustellen.

\section{Diskussion}

Die Daten der DEGS1-Studie sprechen dafür, dass nach wie vor viele Erwachsene in Deutschland rauchen. Bezo- gen auf die 18- bis 79-Jährigen rauchen $26,9 \%$ der Frauen täglich oder gelegentlich, von den Männern sind es 32,6\%. Am stärksten verbreitet ist das Rauchen bei jungen Erwachsenen im Alter von 18 bis 29 Jahren. Auch im mittleren Lebensalter, zwischen dem 30. und 64. Lebensjahr, erfährt das Rauchen einen großen Zuspruch. Ein deutlicher Rückgang des Rauchens ist erst nach dem 65. Lebensjahr festzustellen. Dieser Rückgang dürfte nicht allein mit Änderungen der Le- bensbedingungen und der Lebensweise nach dem Ausscheiden aus dem Erwerbsleben zusammenhängen, sondern ist auch vor dem Hintergrund des vermehrten Auftretens von tabakass oziierten Erkrankungen und vorzeitigen Todesfällen zu sehen [20].

Bezüglich des starken Rauchens, das an einem Konsum von 20 Zigaretten und mehr am Tag festgemacht wurde, zeigt sich eine andere Verteilung über die betrachteten Altersgruppen. Die höchsten Prävalenzen sind bei Frauen wie Männern im mittleren Lebensabschnitt festzustellen. Die Zunahme des Anteils der starken Raucherinnen und Raucher vom jungen zum mittleren Lebensalter wird darauf zurückgeführt, dass mit der Dauer des Rauchens aufgrund der einsetzenden Gewöhnung eine höhere Nikotinzufuhr erfolgen muss, um die psychotrope Wirkung zu erzielen [21]. Dass der Anteil der starken Raucherinnen und Raucher nach dem 65 . Lebensjahr zurückgeht, ist unter anderem dem erhöhten Erkrankungs- und vorzeitigen Sterberisiko von starken Raucherinnen und Rauchern geschuldet [22].

Darüber hinaus sprechen die Daten der DEGS1-Studie für einen Zusammenhang zwischen dem sozialen Status und dem Rauchverhalten. Diese Unterschiede, die zuungunsten der niedrigen und auch der mittleren im Vergleich zur hohen Statusgruppe ausfallen, wurden bereits in zahlreichen anderen Studien berichtet. Neben Analysen auf Basis der früheren Gesundheitssurveys des Robert Koch-Instituts $[23,24]$ ist auf Studien anhand von Daten des Mikrozensus des Statistischen Bundesamtes [25] und des Sozio-oekonomischen Panels des Deutschen Instituts für Wirtschaftsforschung [26] zu verweisen.

Die Ergebnisse der DEGS1-Studie, die sich auf das Jemalsrauchen und die Ausstiegsquote beziehen, sprechen dafür, dass mit Blick auf die betrachteten Altersjahrgänge die statusspezifischen Unterschiede im Rauchverhalten vor allem darauf zurückzuführen sind, dass Personen mit niedrigem Sozialstatus seltener mit dem Rauchen wieder aufhören (vgl. [20]). Bei Männern, nicht jedoch bei Frauen, kommt hinzu, dass von den Angehörigen der niedrigen Statusgruppe ein 


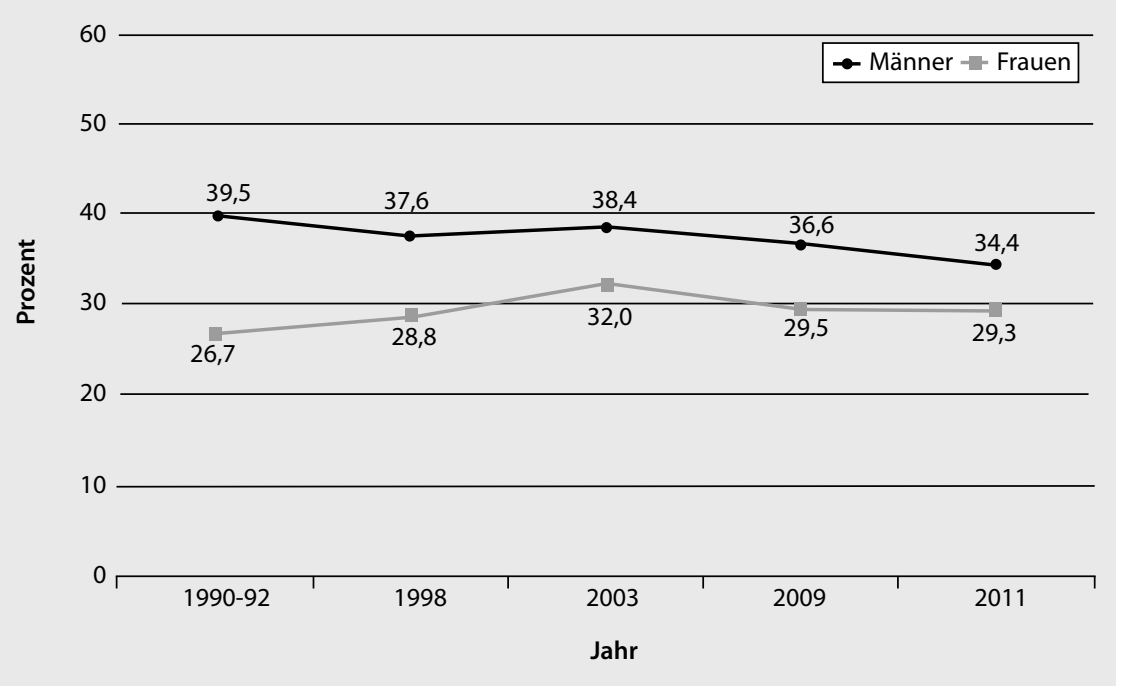

Abb. $4 \triangle$ Zeitliche Entwicklung des Anteils der Raucherinnen und Raucher in der 25- bis 69-jährigen Bevölkerung. Dargestellt sind Prävalenzen (in \%) ( $n=7466$ für NUS90-92, $n=5825$ für BGS98, $n=6890$ für GSTel03, $n=16.418$ für GEDA09, $n=7272$ für DEGS1)

größerer Anteil mit dem Rauchen angefangen hat.

Der Vergleich mit den früheren Gesundheitssurveys deutet darauf hin, dass der Anteil der Raucherinnen und Raucher in den letzten 10 Jahren etwas zurückgegangen ist. Die Veränderungen im Zeitraum von 2003 bis 2011 können für die 25- bis 79-jährige Bevölkerung als statistisch signifikant erachtet werden. Bei einer für Alter und Geschlecht differenzierten Betrachtung erweist sich der Rückgang bei Männern in allen Altersgruppen als statistisch bedeutsam mit Ausnahme der 45- bis 64-Jährigen. Bei Frauen lässt sich nur der Rückgang in der Altersgruppe der 30- bis 44-Jährigen statistisch absichern.

Bei der Einordnung der Ergebnisse zu den Veränderungen des Rauchverhaltens über die Zeit müssen die im Methodenabschnitt angesprochenen Unterschiede im Studiendesign und in der Stichprobenzusammensetzung berücksichtigt werden. Entsprechend vorsichtig sollte der Rückgang des Rauchens bewertet werden, zumal er sich erst in den letzten Jahren abzeichnet. Die nachfolgenden Gesundheitssurveys müssen zeigen, ob sich diese Entwicklung weiter fortsetzt. Ebenso lassen die vorliegenden Ergebnisse keinen unmittelbaren Rückschluss darauf zu, inwieweit die politischen Maßnahmen zur Eindämmung des Tabakkonsums erfolgreich gewesen sind. Auffällig rolle der Maßnahmen und Programme von Bedeutung, insbesondere wenn diese unter Einbindung der relevanten Ak- teure formuliert werden und mit konkreten Handlungsempfehlungen verknüpft sind $[29,30]$.

\section{Korrespondenzadresse}

\section{Dr. T. Lampert}

Abteilung für Epidemiologie und Gesundheitsmonitoring, Robert Koch-Institut General-Pape-Str. 62-66, 12101 Berlin t.lampert@rki.de

Finanzierung der Studie. Die Studie wurde finanziert mit Mitteln des Robert Koch-Instituts und des Bundesministeriums für Gesundheit.

Interessenkonflikt. Der korrespondierende Autor gibt für sich und seine Koautoren an, dass kein Interessenkonflikt besteht.

\section{Literatur}

1. International Agency for Research on Cancer (2004) IARC Monographs on the evaluation of the carcinogenic risks to humans. Tobacco smoke and involuntary smoking. International Agency for Research on Cancer, Lyon

2. US Department of Health and Human Services (2004) The health consequences of smoking: a report of the surgeon general. US Department of Health and Human Services, Centers for Disease Control and Prevention, National Center for Chronic Disease Prevention and Health Promotion, Office on Smoking and Health. USDHHS, Atlanta, Georgia

3. Deutsches Krebsforschungszentrum (DKFZ) (2009) Tabakatlas Deutschland 2009. DKFZ, Heidelberg. http://www.dkfz.de/de/tabakkontrolle/download/Publikationen/sonstVeroefentlichungen/Tabakatlas_2009.pdf (Zugegriffen: 31.10.2012)

4. Mons U (2011) Tabakattributable Mortalität in Deutschland und in den deutschen Bundesländern - Berechnungen mit Daten des Mikrozensus und der Todesursachenstatistik. Gesundheitswesen 73:238-246

5. Deutsches Krebsforschungszentrum (DKFZ) (2010) Schutz der Familie vor Tabakrauch. Rote Reihe Tabakprävention und Tabakkontrolle Bd 14. DKFZ, Heidelberg. http://www.dkfz.de/de/tabakkontrolle/download/Publikationen/RoteReihe/ Band_14_Schutz_der_Familie_vor_Tabakrauch. pdf (Zugegriffen: 31.10.2012)

6. Neubauer S, Welte R, Beiche A et al (2006) Mortality, morbidity and costs attributable to smoking in Germany: update and a 10-year comparison. Tob Control 15:464-471

7. Drogenbeauftrage der Bundesregierung (2011) Drogen- und Suchtbericht. Die Drogenbeauftrage der Bundesregierung, Berlin

8. Kurth BM, Lange C, Kamtsiuris P, Hölling H (2009) Gesundheitsmonitoring am Robert Koch-Institut, Sachstand und Perspektiven. Bundesgesundheitsbl Gesundheitsforsch Gesundheitsschutz 52:557-570

9. Kurth BM (2012) Das RKI-Gesundheitsmonitoring - was es enthält und wie es genutzt werden kann. Public Health Forum 76:4.e1-4.e3 
10. Gößwald A, Lange M, Kamtsiuris P, Kurth BM (2012) DEGS: Studie zur Gesundheit Erwachsener in Deutschland. Bundesweite Quer- und Längsschnittstudie im Rahmen des Gesundheitsmonitorings des Robert Koch-Instituts. Bundesgesundheitsbl Gesundheitsforsch Gesundheitsschutz 55:775-780

11. Scheidt-Nave C, Kamtsiuris P, Gößwald A et al (2012) German Health Interview and Examination Survey for Adults (DEGS) - design, objectives and implementation of the first data collection wave (DEGS1). BMC Public Health 12:730

12. Kamtsiuris $P$, Lange $M$, Hoffmann R et al (2013) Die erste Welle der Studie zur Gesundheit Erwachsener in Deutschland (DEGS1): Stichprobendesign, Response, Gewichtung und Repräsentativität. Bundesgesundheitsbl Gesundheitsforsch Gesundheitsschutz 56:620-630

13. Robert Koch-Institut (Hrsg) (2009) DEGS: Studie zur Gesundheit Erwachsener in Deutschland Projektbeschreibung. Beiträge zur Gesundheitsberichterstattung des Bundes. RKI, Berlin

14. Gößwald A, Lange M, Dölle R, Hölling H (2013) Die erste Welle der Studie zur Gesundheit Erwachsener in Deutschland (DEGS1). Gewinnung von Studienteilnehmenden, Durchführung der Feldarbeit und Qualitätsmanagement. Bundesgesundheitsbl Gesundheitsforsch Gesundheitsschutz 56:611619

15. Lampert T, Kroll LE, Müters S, Stolzenberg H (2013) Messung des sozioökonomischen Status in der Studie zur Gesundheit Erwachsener in Deutschland (DEGS). Bundesgesundheitsbl Gesundheitsforsch Gesundheitsschutz 56:631-636
16. Lampert T, Kroll LE, Müters S, Stolzenberg H (2013) Messung des sozioökonomischen Status in der Studie "Gesundheit in Deutschland aktuell" 2009. Bundesgesundheitsbl Gesundheitsforsch Gesundheitsschutz 56(1):131-143

17. Latza U, Hoffmann W, Terschüren C et al (2005) Erhebung, Quantifizierung und Analyse der Rauchexposition in epidemiologischen Studien. Robert Koch-Institut, Berlin

18. Robert Koch-Institut (Hrsg) (2009) Daten und Fakten: Ergebnisse der Studie "Gesundheit in Deutschland aktuell 2009". Beiträge zur Gesundheitsberichterstattung des Bundes. RKI, Berlin

19. Kroll L (2010) Sozialer Wandel, soziale Ungleichheit und Gesundheit. Die Entwicklung sozialer und gesundheitlicher Ungleichheit in Deutschland zwischen 1984 und 2006. VS Verlag für Sozialwissenschaften, Wiesbaden

20. Lampert T, Burger M (2004) Rauchgewohnheiten in Deutschland - Ergebnisse des telefonischen Gesundheitssurveys 2003. Gesundheitswesen 66:511-517

21. Lampert T (2011) Rauchen - Aktuelle Entwicklungen bei Erwachsenen. GBE kompakt 4/11. http:// edoc.rki.de/series/gbe-kompakt/2011-9/PDF/9. pdf (Zugegriffen: 31.10.2012)

22. Jha $P$, Ramasundarahettige $C$, Landsman $V$ et al (2013) 21st-Century hazards of smoking and benefits of cessation in the United States. N Engl J Med 368(4):341-350

23. Lampert T, Thamm M (2004) Soziale Ungleichheit des Rauchverhaltens in Deutschland. Bundesgesundheitsbl Gesundheitsforsch Gesundheitsschutz 47:1033-1042
24. Nocon M, Keil T, Willich S (2007) Education, income, occupational status and health risk behaviour. J Public Health 15(5):401-405

25. Helmert U, Borgers D, Bammann K (2001) Soziale Determinanten des Rauchverhaltens in Deutschland: Ergebnisse des Mikrozensus 1995. Sozial Präventivmed 46:172-181

26. Lampert $\mathrm{T}$ (2010) Soziale Determinanten des Tabakkonsums bei Erwachsenen in Deutschland. Bundesgesundheitsbl Gesundheitsforsch Gesundheitsschutz 53(2/3):108-116

27. Maschewsky-Schneider U, Pott E (2010) Tabakprävention in Deutschland. Bundesgesundheitsbl Gesundheitsforsch Gesundheitsschutz 53:89-90

28. Mons U, Pöttschke-Langer M (2010) Gesetzliche Maßnahmen zur Tabakprävention. Evidenz, Erfolge und Barrieren. Bundesgesundheitsbl Gesundheitsforsch Gesundheitsschutz 53:144-151

29. Kröger C, Mons U, Klärs G et al (2010) Evaluation des Gesundheitsziels „Tabakkonsum reduzieren“. Bundesgesundheitsbl Gesundheitsforsch Gesundheitsschutz 53:91-102

30. BMG - Bundesministerium für Gesundheit (2003) gesundheitsziele.de - Forum zur Entwicklung und Umsetzung von Gesundheitszielen in Deutschland. BMG, Berlin

\section{Hier steht eine Anzeige.}

